\title{
ARTICLE OPEN RIOX1-demethylated cGAS regulates ionizing radiation-elicited DNA repair
}

Yanxuan Xiao ${ }^{1}$, Jingyi $\mathrm{Li}^{2,3}$, Xiaoyu Liao ${ }^{1}$, Yumin $\mathrm{He}^{1}$, Tao He${ }^{4}$, Cuiping Yang ${ }^{5}$, Lu Jiang ${ }^{1}$, So Mi Jeon ${ }^{6}$, Jong-Ho Lee ${ }^{6,7}$, Yongbin Chen ${ }^{5,8}$, Rui Liu ${ }^{1 凶}$ and Qianming Chen ${ }^{1 凶}$

Exposure to radiation causes DNA damage; hence, continuous surveillance and timely DNA repair are important for genome stability. Epigenetic modifications alter the chromatin architecture, thereby affecting the efficiency of DNA repair. However, how epigenetic modifiers coordinate with the DNA repair machinery to modulate cellular radiosensitivity is relatively unknown. Here, we report that loss of the demethylase ribosomal oxygenase 1 (RIOX1) restores cell proliferation and reduces cell death after exposure to ionizing radiation. Furthermore, RIOX1 depletion enhances homologous recombination (HR) repair but not nonhomologous endjoining (NHEJ) repair in irradiated bone marrow cells and oral mucosal epithelial cells. Mechanistic study demonstrates that RIOX1 removes monomethylation at K491 of cyclic GMP-AMP synthase (cGAS) to release cGAS from its interaction with the methyl-lysine reader protein SAGA complex-associated factor 29 (SGF29), which subsequently enables cGAS to interact with poly(ADP-ribosyl) ated poly(ADP-ribose) polymerase 1 (PARP1) at DNA break sites, thereby blocking PARP1-mediated recruitment of Timeless. High expression of RIOX1 maintains CGAS K491me at a low level, which impedes HR repair and reduces cellular tolerance to ionizing radiation. This study highlights a novel RIOX1-dependent mechanism involved in the non-immune function of cGAS that is essential for the regulation of ionizing radiation-elicited HR repair.

Bone Research (2022)10:19 ～～～https://doi.org/10.1038/s41413-022-00194-0

\section{INTRODUCTION}

Exposure to ionizing radiation may cause both acute symptoms and long-term health effects. ${ }^{1}$ Among mammalian organs, the bone marrow is the most vulnerable to ionizing radiation-induced damage due to the rapid turnover of immature hematopoietic cells. While suppression of mature blood cells is a common consequence in patients who receive radiotherapy, mortality is believed to be caused by the exhaustion of both hematopoietic progenitor cells and primitive hematopoietic stem cells in the bone marrow. ${ }^{2}$

lonizing radiation results in various lesions in both genomic and mitochondrial DNA, among which double-strand breaks (DSBs) are arguably the most harmful. ${ }^{3}$ DSBs are repaired via two main pathways, homologous recombination (HR) and nonhomologous end-joining (NHEJ). ${ }^{4}$ HR repair of damaged sites involves template-guided DNA extension, which guarantees error-free repair by restoring sequence information from the templates. In contrast, in NHEJ repair, the two broken ends of chromosomes are directly ligated in an error-prone manner. ${ }^{5}$

Jumonji C domain (JMJD)-containing demethylase family, which includes over 30 members, plays an important role in regulating DNA repair. $^{6-8}$ Ribosomal oxygenase 1 (RIOX1) removes mono- or trimethylation from histone 3 lysine 4 (H3K4me1 or H3K4me3, respectively), while it has weaker activity toward methylated H3K36. ${ }^{9}$ However, whether RIOX1 is involved in DNA repair upon ionizing radiation-induced damage is largely undefined. In the current study, we report a novel RIOX1mediated mechanism that modulates the efficiency of HR repair. We show that depletion of RIOX1 in mouse bone marrow cells restores cell viability and decreases cell death upon exposure to ionizing radiation. Loss of RIOX1 enhances HR repair but not NHEJ repair in irradiated cells. Furthermore, we demonstrate that RIOX1 abolishes the monomethylation of K491 in cyclic GMPAMP synthase (cGAS) (corresponding to K506 in human cGAS), which disrupts the interaction between CGAS and the methyllysine reader protein SAGA complex-associated factor 29 (SGF29). cGAS without K491me binds to poly(ADP-ribosyl)ated poly(ADPribose) polymerase 1 and abrogates the recruitment of Timeless to DNA break sites, thereby blocking HR repair. RIOX1 is highly expressed in bone marrow cells with a relatively low level of cGAS K491me, consistent with the high radiosensitivity of bone marrow cells.

\footnotetext{
${ }^{1}$ State Key Laboratory of Oral Diseases, National Clinical Research Center for Oral Diseases, Chinese Academy of Medical Sciences Research Unit of Oral Carcinogenesis and Management, West China Hospital of Stomatology, Sichuan University, Chengdu, Sichuan 610041, China; ${ }^{2}$ The Second Affiliated Hospital of Chengdu Medical College, China National Nuclear Corporation 416 Hospital, Chengdu, Sichuan 610051, China; ${ }^{3}$ School of Biological Sciences and Technology, Chengdu Medical College, Chengdu 610599, China; ${ }^{4}$ Department of Cardio-thoracic Surgery, the Second Affiliated Hospital of Chengdu Medical College, China National Nuclear Corporation 416 Hospital, Chengdu, Sichuan 610051 , China; ${ }^{5}$ Key Laboratory of Animal Models and Human Disease Mechanisms of Chinese Academy of Sciences Yunnan Province, Kunming Institute of Zoology, Kunming, Yunnan

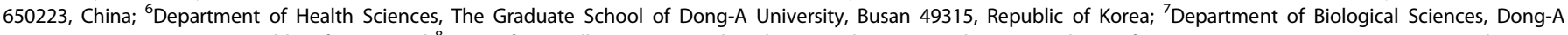
University, Busan 49315, Republic of Korea and ${ }^{8}$ Center for Excellence in Animal Evolution and Genetics, Chinese Academy of Sciences, Kunming, Yunnan 650223 , China Correspondence: Rui Liu (liurui_scu@hotmail.com) or Qianming Chen (qmchen@scu.edu.cn)

These authors contributed equally: Yanxuan Xiao, Jingyi Li
}

Received: 24 April 2021 Accepted: 10 August 2021

Published online: 24 February 2022 
a
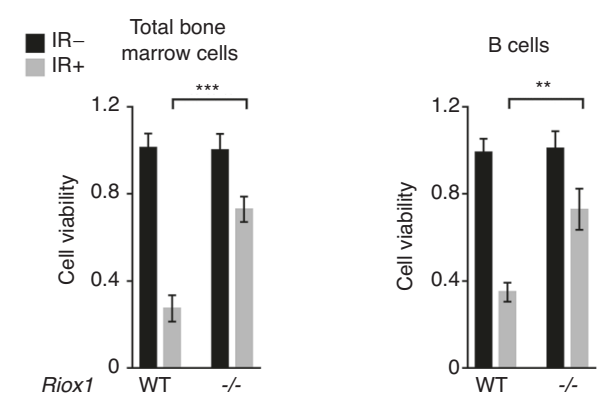

b
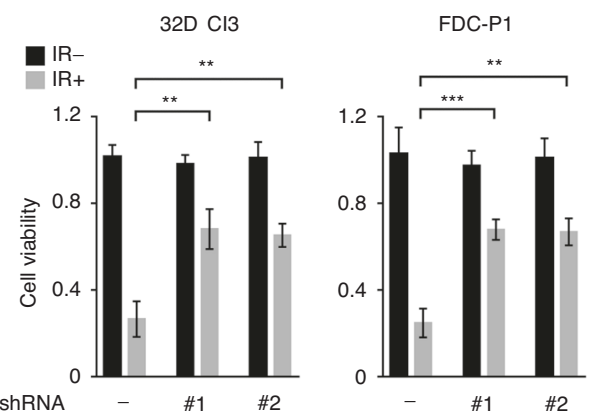

d Riox1 shRNA \#1
Riox1 shRNA \#2
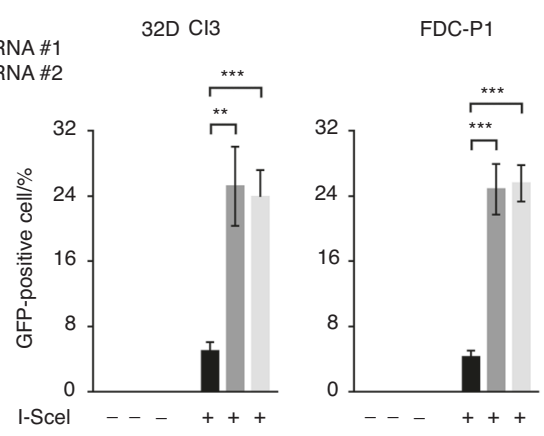

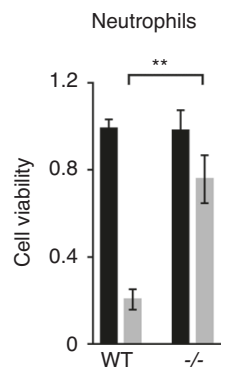

C
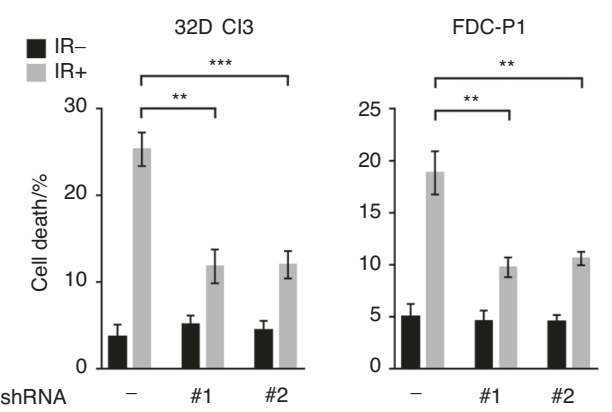

e
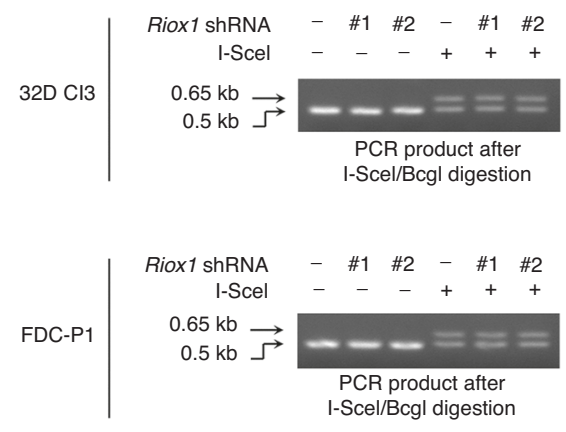

Fig. 1 RIOX 1 decreases cell viability and inhibits HR repair in bone marrow cells after ionizing radiation. The data are shown as the mean \pm SD of three independent assays. ${ }^{* *} P<0.01 ;{ }^{* *} P<0.001$. a WT or Riox $1^{-1-}$ mice were treated with 9 Gy ionizing radiation. The indicated bone marrow cells were isolated $16 \mathrm{~h}$ after irradiation, and cell viability was measured. b, c 32D Cl3 and FDC-P1 cells expressing Riox 1 shRNAs were treated with $10 \mathrm{~Gy}$ ionizing radiation. Cell viability (b) and cell death (c) were evaluated $48 \mathrm{~h}$ after irradiation. Cell viability data were normalized to the untreated group. 32D Cl3 and FDC-P1 cells expressing Riox 1 shRNAs were transfected with an I-Scel expression vector. HR repair was tested $72 \mathrm{~h}$ after transfection (d). NHEJ repair was examined $1 \mathrm{~h}$ after irradiation (e)

\section{RESULTS}

RIOX1 decreases cell viability and inhibits HR repair in bone marrow cells after ionizing radiation

To evaluate the effects of RIOX1 on bone marrow cells after ionizing radiation, RIOX1 knockout mice were examined. Adult RIOX1 knockout mice were viable and fertile. We noted an increased body weight and length at birth and at 8 weeks of age (Fig. S1A and S1B). Loss of RIOX1 resulted in prolonged survival (Fig. S1C) and attenuated symptoms of diarrhea (Fig. S1D) after irradiation. We further obtained bone marrow cells from the tibiae of the irradiated mice and ultimately isolated B cells, hematopoietic progenitor cells (HPCs) and neutrophils. As expected, ionizing radiation largely decreased the viability of total bone marrow cells and all three cell subsets tested (Fig. 1a). Notably, the irradiation-induced decrease in cell viability was substantially reversed in bone marrow cells isolated from RIOX1 knockout mice compared to those isolated from their wild-type (WT) counterparts (Fig. 1a). Furthermore, we treated the mouse bone marrow myeloid 32D Cl3 cell line, which expresses CD11a and $C D 11 b^{10}$ but not the fibroblast marker vimentin or epithelial cell marker E-Cad (Fig. S1E), with $10 \mathrm{~Gy}$ ionizing radiation.
Consistent with the above findings, knockdown of endogenous RIOX1 in 32D Cl3 cells by two distinct shRNAs substantially attenuated the irradiation-induced cell viability suppression and cell death (Fig. $1 b$ and $c$ ). Similar results were obtained in the CD34- and Thy-1-expressing mouse bone marrow myeloid cell line FDC-P $1^{11}$ (Figs. $1 \mathrm{~b}, \mathrm{c}$ and S1E).

Effective DNA repair is important to fix DNA damage. ${ }^{12}$ Strikingly, knockout of RIOX1 largely eliminated the irradiationinduced DNA fragments in bone marrow cells, as revealed by the shortened tail-like smear in the comet assay (Fig. S1F), suggesting that RIOX1 is likely involved in modulating irradiation-elicited DNA damage. To determine the impact of RIOX1 on HR and NHEJ repair, the endonuclease I-Scel was expressed to generate a DSB site in an exogenously introduced DR-GFP locus. DNA repair through HR removed the I-Scel cut site and generated a Bcgl cut site, leading to the expression of DR-GFP; in contrast, NHEJ repair resulted in a $0.65-\mathrm{kb}$ PCR product that was resistant to both I-Scel and Bcgl digestion (Fig. S1G). ${ }^{13}$ We found that expression of I-Scel induced GFP expression in approximately 5\% of 32D Cl3 and FDCP1 cells, and this GFP expression was appreciably enhanced upon RIOX1 knockdown (Figs. 1d and $\mathrm{S} 1 \mathrm{H}$ ). The different amounts of 
GFP-expressing cells were presumed to reflect the different HR repair efficiencies, since a similar I-Scel cleavage efficiency was detected in Riox1 shRNA-treated and untreated cells before DNA repair was initiated (Fig. S1I). However, loss of RIOX1 minimally affected NHEJ repair, as revealed by the comparable amounts of the $0.65-k b$ PCR product (Fig. 1e). These results suggest that RIOX1 decreases cell viability and inhibits HR repair in bone marrow cells after ionizing radiation.

RIOX1 binds to cGAS and demethylates cGAS K491me

To explore the mechanisms underlying the RIOX1-mediated repression of HR repair, we depleted RIOX1 activity in 32D Cl3 cells by replacing endogenous WT RIOX 1 with the shRNA-resistant Flag-RIOX1 H302 A/H367A mutant (corresponding to human RIOX1 H339A/H404A), which lacks lysine demethylase activity (Fig. S2A). ${ }^{9}$ Loss of RIOX1 activity induced an increase in HR repair efficiency similar to that caused by RIOX1 knockdown (Fig. S2A), suggesting that demethylase activity is required for RIOX1mediated repression of HR repair. Surprisingly, reconstitution of RIOX1 expression with this mutant only evoked limited accumulation of H3K4me, H3K4me3 and H3K36me3 (Fig. S2B), which might be interpreted in the context of previous reports showing that methylation of these sites can also be removed by a couple of other lysine demethylases. ${ }^{14}$ Furthermore, human histone $\mathrm{H} 3$ is encoded by several genes and expressed as multiple variants, which are highly conserved with only a few nonconserved residues. $^{15}$ In line with previous reports, ${ }^{16,17}$ targeting $\mathrm{H} 3.3 \mathrm{~B}$ mRNA (gene symbol: $\mathrm{H} 3 \mathrm{f} 3 \mathrm{~b}$ ) reduced total $\mathrm{H} 3$ expression by approximately $80 \%$ in $32 \mathrm{D} \mathrm{Cl} 3$ cells, as revealed by analysis with an antibody that recognizes all $\mathrm{H} 3$ variants, suggesting that $\mathrm{H} 3.3 \mathrm{~B}$ is the major histone $\mathrm{H} 3$ isoform (Fig. S2C). Strikingly, reconstitution of $\mathrm{H} 3.3 \mathrm{~B}$ expression with the shRNA-resistant H3.3B K4R or K36R mutant failed to abolish the increased HR repair in RIOX1-depleted $32 \mathrm{D} \mathrm{Cl} 3$ cells (Fig. S2C). Together, these results suggest that a nonhistone substrate is likely involved in RIOX1-mediated regulation of HR repair.

Next, we pulled down Flag-tagged RIOX1 protein from 32D Cl3 cells, and cGAS was detected in the RIOX1 immunoprecipitates by mass spectrometry (Fig. S3A). cGAS was previously found to translocate into the nucleus upon ionizing radiation and block HR repair. ${ }^{18}$ The interaction between endogenous RIOX 1 and cGAS was confirmed by coimmunoprecipitation in both 32D Cl3 and FDC-P1 cells (Fig. 2a). An in vitro protein binding assay showed that bacterially purified cGAS protein could be pulled down by purified GST-RIOX1 protein, suggesting that these two proteins directly interact with each other (Fig. 2b). However, no interaction between CGAS and RIOX2, which shares $40 \%$ homology with RIOX1, was detected (Fig. 2a). Furthermore, reconstitution of cGAS expression with the cGAS Y201E mutant (corresponding to human cGAS $\mathrm{Y} 215 \mathrm{E})$, which is resistant to ionizing radiation-induced translocation into the nucleus, ${ }^{18}$ enhanced $\mathrm{HR}$ repair in $32 \mathrm{D} \mathrm{Cl} 3$ cells, and HR repair could not be further increased by RIOX1 knockdown (Fig. 2c). In contrast, overexpression of cGAS substantially reduced RIOX1 depletion-induced HR repair (Fig. 2d).

To determine whether RIOX1 affects the methylation status of cGAS, we analyzed cGAS protein extracted from RIOX1-depleted 32D Cl3 cells and identified monomethylation of cGAS K491, which is evolutionarily conserved among species (corresponding to human cGAS K506, Fig. S3B). Additionally, the residues adjacent to K491 are analogous to those adjacent to H3K4 or and H3K36 (Fig. S3B). To quantify this methylation, we produced an antibody that specifically recognizes the mouse cGAS K491me peptide but not the unmodified K491, K491me2 or K491me3 peptide (Fig. S3C). By using this antibody, we found that cGAS K491me highly accumulated in cells with depletion of RIOX1 expression or activity (Fig. 2e and $f$ ), although the expression level of endogenous CGAS was unchanged (Fig. 2e and f). In contrast, knockdown of JMJD1A, JMJD1B, or LSD1, all of which are capable of removing H3K4me or
H3K36me, did not affect the level of cGAS K491me in 32D Cl3 cells (Fig. S3D), suggesting that these enzymes are not responsible for cGAS K491me demethylation. Immunoblotting showed that this modification was abolished by incubation with an excess of the cGAS K491me peptide, suggesting the good specificity of the anticGAS K491me antibody (Fig. S3E). Consistent with this finding, overexpression of RIOX 1 or the CGAS K491R mutant abrogated this modification (Figs. $2 \mathrm{~g}$ and $\mathrm{h}$ ). However, ionizing radiation had minor effects on the level of cGAS K491me in both RIOX1expressing and RIOX1-depleted cells (Fig. S3F).

To verify whether RIOX1 directly catalyzes the demethylation of cGAS K491me, Flag-cGAS protein was purified from RIOX1depleted 32D Cl3 cells and was then subjected to an in vitro demethylation assay. The K491me modification was clearly detected in the purified cGAS protein (Fig. 2i). Moreover, incubation with bacterially purified WT RIOX1 protein but not the purified RIOX1 H302A/H367A mutant or the RIOX2 protein resulted in erasure of cGAS K491me (Fig. 2i). These results suggest that RIOX1 binds to and demethylates CGAS K491me.

\section{RIOX1 overrides SET7-mediated methylation and governs cGAS K491me}

We sought to identify the potential methyltransferases responsible for cGAS K491me by screening a set of reported enzymes. Knockdown of only SET domain-containing lysine methyltransferase 7 (SET7) reduced the cellular level of cGAS K491me (Fig. 3a). This observation was confirmed by treating 32D Cl3 and FDC-P1 cells with two distinct Set7 shRNAs (Fig. 3b). SET7 protein was found in the CGAS immunoprecipitates, indicating the interaction between endogenous SET7 and cGAS (Fig. 3c). Furthermore, an in vitro protein methylation assay revealed that incubation with bacterially purified His-SET7 protein induced K491me of GSTcGAS, while this methylation was blocked when the methylatable lysine-substituted CGAS K491R mutant or the catalytically dead SET7 H297A mutant was used in the reaction system (Fig. 3d, e). These results suggest that SET7 methylates cGAS at K491.

To determine how RIOX1 and SET7 mutually regulate cGAS K491me in cells, we compared the level of cGAS K491me and the expression of RIOX1 and SET7 among various mouse organs. We found that, compared to other organs, the bone marrow showed an increased expression level of RIOX1 and a reduced level of cGAS K491me (Fig. 3f). However, SET7 was ubiquitously expressed in different organs, with minor changes (Fig. 3f). Consistent with this finding, RIOX1 expression was enriched in 32D Cl3 cells, FDC$P 1$ cells and mouse bone marrow-derived B cells and neutrophils with a decreased level of cGAS K491me; in contrast, lower RIOX1 expression level and a correspondingly increased cGAS K491me level were found in the normal mouse liver cell line AML12, lung epithelial cell line MLE 12, myoblast line C2C12 and fibroblast line $\mathrm{NIH} / 3 \mathrm{~T} 3$ (Fig. $3 \mathrm{~g}$ ). These results suggest that RIOX1 expression governs the level of cGAS K491me.

RIOX1-mediated demethylation of cGAS K491me facilitates cGAS binding to PARP1

cGAS recognizes double-stranded DNA, including endogenous genomic DNA fragments, to activate innate immune responses. ${ }^{19}$ However, loss of RIOX1 has limited impact on ionizing radiationinduced phosphorylation of TANK-binding kinase 1 (TBK1) at S172 (Fig. S4A), a hallmark of cGAS activation ${ }^{20}$. This finding was further supported by the observation that herring testis (HT)-DNA equally elicited the catalytic activity of purified CGAS protein with or without SET7-mediated K491me (Fig. S4B). Furthermore, the cellular fractionation assay showed a similar subcellular distribution of cGAS in untreated and Riox 1 shRNA-treated cells (Fig. S4C), suggesting that RIOX1 does not regulate the cytoplasmic-nuclear shuttling of cGAS.

lonizing radiation-induced nuclear cGAS interacts with PARP1 by binding with poly(ADP ribose) (PAR) chains and hinders 
a

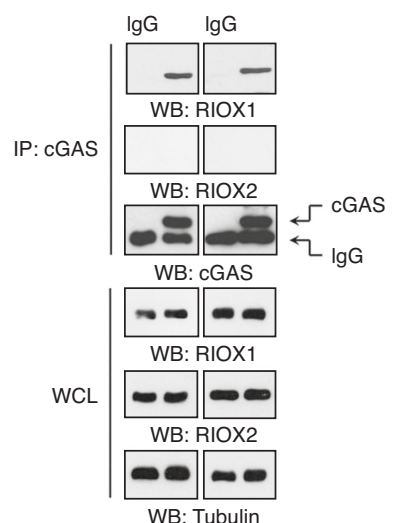

d

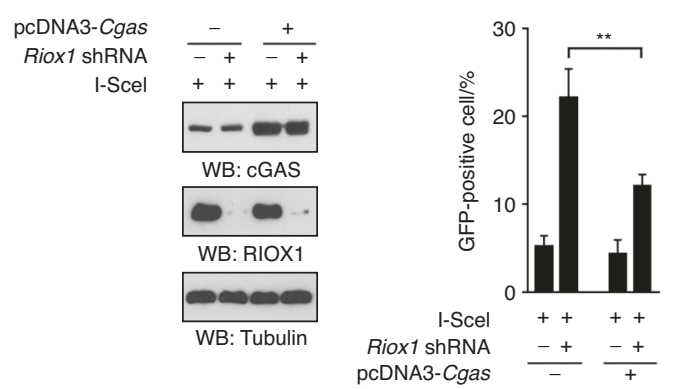

e

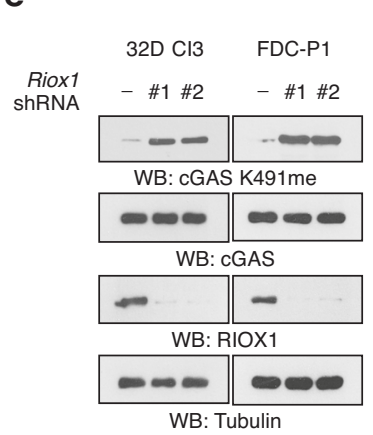

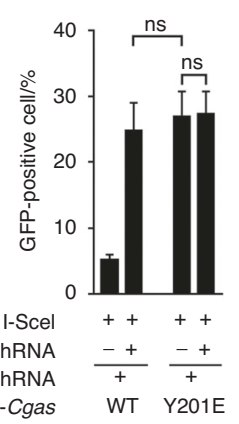

g

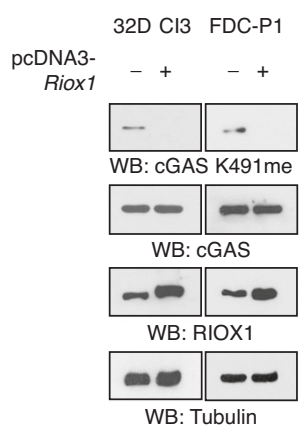

h

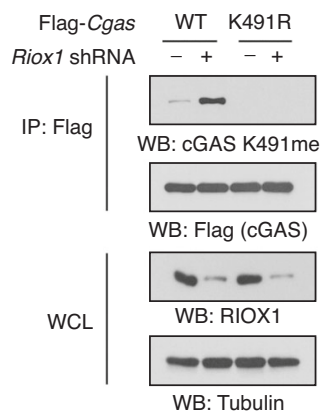

i

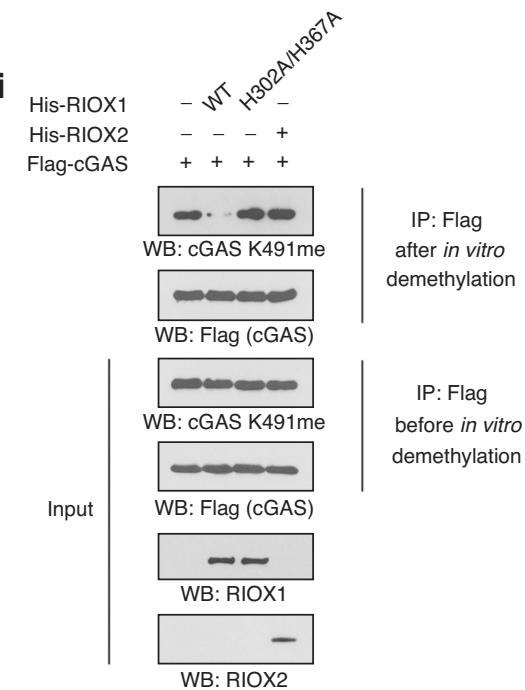

Fig. 2 RIOX1 binds to and demethylates CGAS K491me. a-i Immunoblot analysis was performed. a Coimmunoprecipitation was performed using 32D Cl3 and FDC-P1 cell samples. b Bacterially purified GST-RIOX1 or GST protein was mixed with purified His-cGAS protein. A GST pulldown assay was performed. c 32D Cl3 cells expressing Riox1 shRNA, Cgas shRNA, WT Flag-Cgas, or Flag-Cgas Y201E were transfected with an I-Scel expression vector. HR pathway activity was measured. The Cgas shRNA targeted the noncoding region. The data are shown as the mean \pm SD of three independent assays. ns, not significant. d 32D Cl3 cells expressing Riox1 shRNA or Cgas were transfected with an I-Scel expression vector. HR pathway activity was measured. The data are shown as the mean $\pm \mathrm{SD}$ of three independent assays. ${ }^{* *} P<0.01$. e $32 \mathrm{D} \mathrm{Cl} 3$ and FDC-P1 cells were transduced with Riox 1 shRNAs. $\mathbf{f}$ 32D Cl3 cells were transduced with Riox1 shRNA, WT Flag-Riox1 or Flag-Riox1 H302A/ H367A. The Riox1 shRNA targeted the noncoding region. g 32D CI3 and FDC-P1 cells were transduced with a Riox 1 overexpression vector. h 32D Cl3 cells were transfected with Riox1 shRNA, WT Flag-Cgas, or Flag-Cgas K491R. Immunoprecipitation was performed. i Flag-cGAS protein was purified from Riox1-depleted 32D Cl3 cells and incubated with bacterially purified WT His-RIOX1, His-RIOX1 H302A/H367A, or WT His-RIOX2 protein for an in vitro demethylation assay

PARP1-mediated recruitment of Timeless, thereby inhibiting HR repair. PAR can bind to proteins through a two-section motif that contains a basic amino acid-enriched $\mathrm{N}$-terminus and a cluster of hydrophobic amino acids interspersed with basic residues at the C-terminus. ${ }^{21}$ Analyses of the CGAS protein sequence revealed four potential PAR binding sites: 238KFKRIPRGNPLSHFL252, 265KFRKIIKEEVKEIKDI280, 391KCCRKECLKLMKYLL405 and 483RKSKEFLSKKIEYER497. Replacement of the basic residues in the 

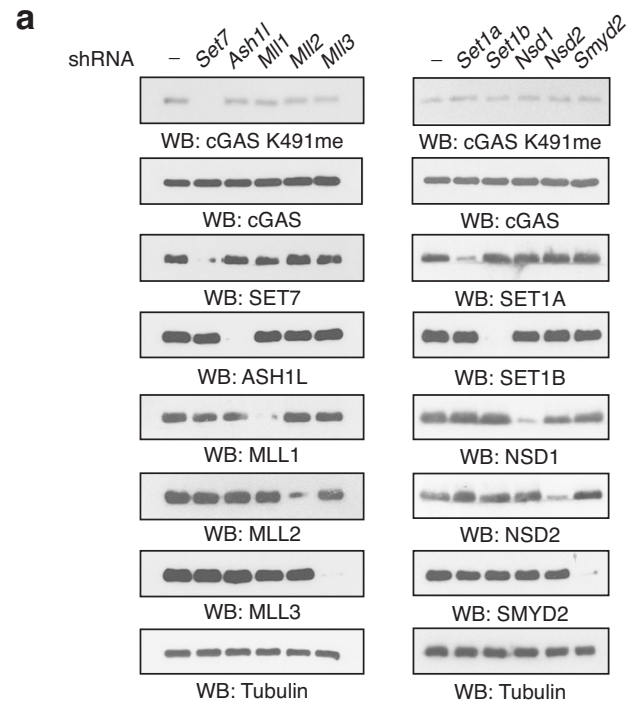

d

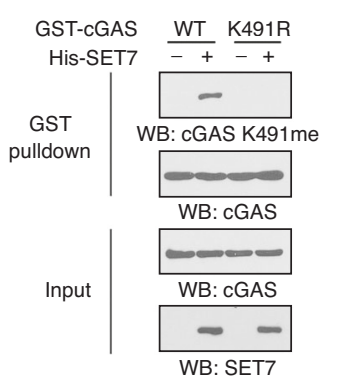

b

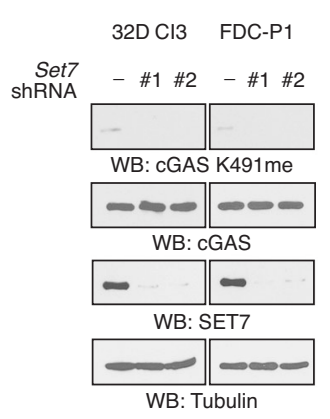

f

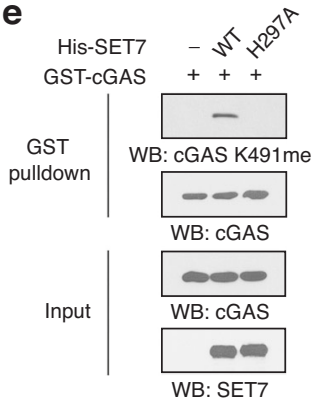

C

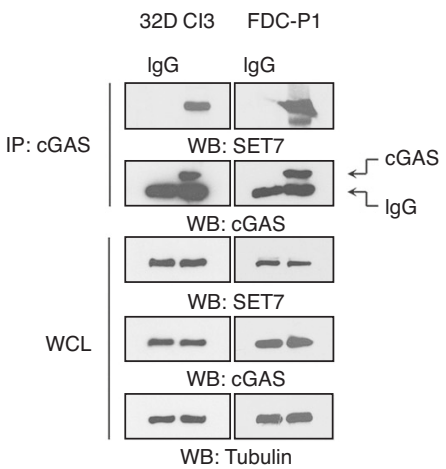

g

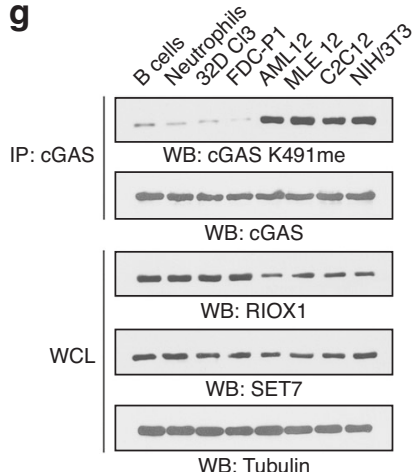

Fig. 3 RIOX1 overrides SET7-mediated methylation and governs CGAS K491 me. a-g Immunoblot analysis was performed. a 32D CI3 cells were transduced with shRNA targeting Set7, Ash11, MII1, MII2, MII3, Set1a, Set1b, Nsd1, Nsd2, or Smyd2. b 32D CI3 and FDC-P1 cells were transduced with Set7 shRNAs. c Coimmunoprecipitation was performed using 32D Cl3 and FDC-P1 cell samples. d Purified WT GST-cGAS or GST-cGAS K491R protein was incubated with purified His-SET7 protein for an in vitro methylation assay. A GST pulldown assay was performed. e Purified GST-cGAS protein was incubated with purified WT His-SET7 or His-SET7 H297A protein for an in vitro methylation assay. A GST pulldown assay was performed. $\mathbf{f}, \mathbf{g}$ Immunoprecipitation was performed using lysates of the indicated mouse tissues or cells

C-termini of these motifs with alanines revealed that only the mutations in the 483-497 aa region reduced the amount of cGAS pulled down by biotin-labeled PAR (Fig. 4a). Furthermore, the 483-497 aa region (corresponding to the 498-512 aa region in humans) did not overlap with the DNA binding region or the catalytic domain of cGAS (Fig. 4b), in line with the observation that the activity of K491-methylated cGAS was inducible by HT-DNA (Fig. S4B).

cGAS K491 is located within the 483-497 aa region, leading to the hypothesis that RIOX1-mediated cGAS K491me may affect cGAS binding with PAR. We performed an in vitro biotin-PAR pulldown assay using the lysates of untreated or RIOX1-depleted $32 \mathrm{D} \mathrm{Cl} 3$ cells that contained equal amounts of cGAS protein. Indeed, equal amounts of biotin-PAR pulled down much less CGAS protein from the lysates of RIOX1-depleted 32D Cl3 cells than from those of untreated 32D Cl3 cells (Fig. 4C), suggesting that RIOX1 facilitates the interaction between cGAS and PAR. A similar result was obtained in the comparison between AML12 and 32D $\mathrm{Cl} 3$ cells, which have different endogenous RIOX1 expression and cGAS K491me levels (Figs. $3 \mathrm{~g}$ and 4c). Furthermore, loss of RIOX1 reduced the amount of cGAS that was either associated with PARP1 in irradiated 32D Cl3 cells or recruited to I-Scel-induced DNA break sites (Figs. 4d, e and S4D). RIOX1 knockdown also promoted the irradiation-elicited translocation of Timeless to DNA break sites and the formation of the PARP1/Timeless complex (Fig. 4e, f). However, these Riox 1 shRNA-mediated effects were largely obliterated by reconstitution of cGAS expression with the
cGAS K491R mutant (Fig. 4g-i). Accordingly, the cGAS K491R mutation also abolished the reinforcement of HR repair in RIOX1depleted cells (Fig. 4j). These results suggest that RIOX1-mediated demethylation of cGAS K491me facilitates cGAS binding to PAR and impedes HR repair.

SGF29 binds to K491me-bearing cGAS and prevents cGAS from binding to PAR

Lysine methylation frequently modulates protein functions via modulating the recruitment of binding partners. ${ }^{22}$ We found that SET7-mediated K491me on the bacterially purified CGAS protein failed to disrupt the interaction between CGAS and PAR, suggesting that other factors are likely involved in this process (Fig. 5a). SGF29, a methyllysine reader protein that can recognize methylated $\mathrm{H} 3 \mathrm{~K}_{4}{ }^{23}$ was found when we revisited the aforementioned proteomic analyses to search for cGAS-associated proteins (Fig. S3A). Loss of RIOX1 increased cGAS K491me and accordingly promoted the interaction of endogenous SGF29 with CGAS in both 32D Cl3 and FDC-P1 cells (Fig. 5b). However, the interaction between these two proteins was abrogated by the cGAS K491R mutation (Fig. 5c) and the SGF29 D194A/D196A mutation, which conferred defective binding to methylated H3K4 (Fig. 5d). ${ }^{24}$

Since cGAS K491 is located within the PAR-binding motif, we reasoned that SGF29 may mask this motif when associated with cGAS, thereby preventing cGAS from binding to PAR. As expected, preincubation with purified His-SGF29 protein markedly abolished 


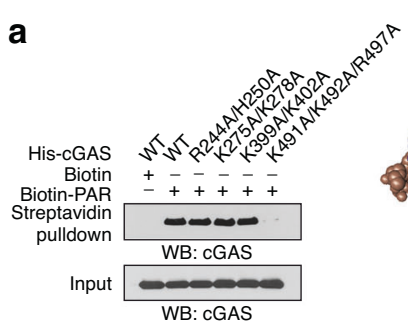

C
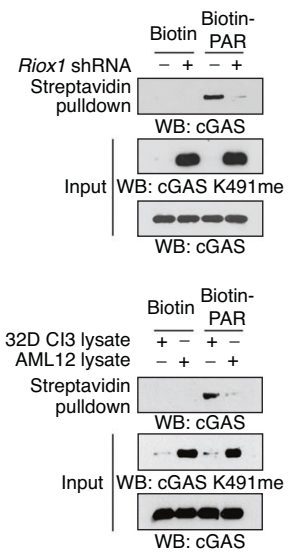

g

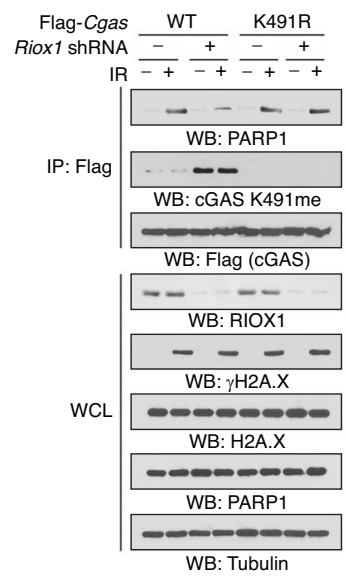

b

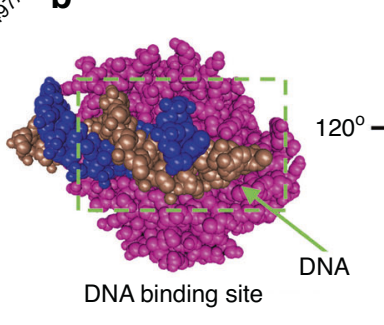

d

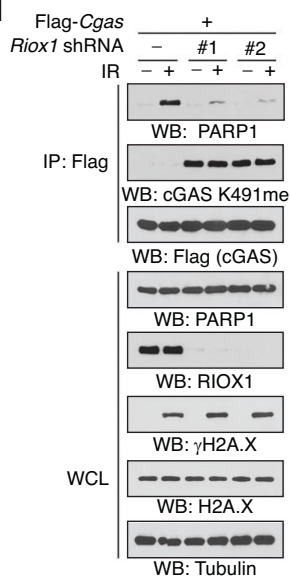

h

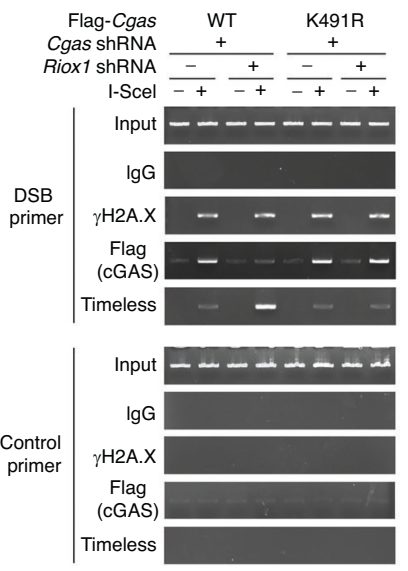

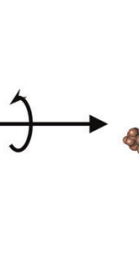
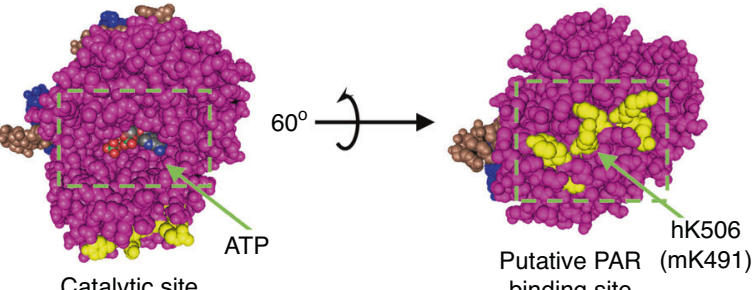

f
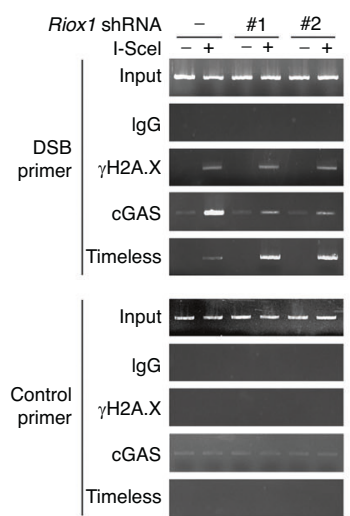

i

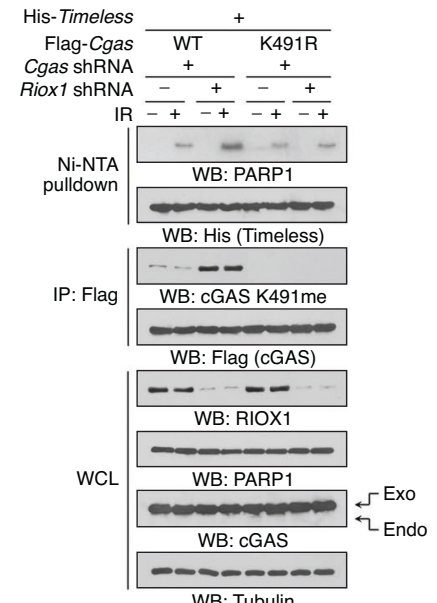

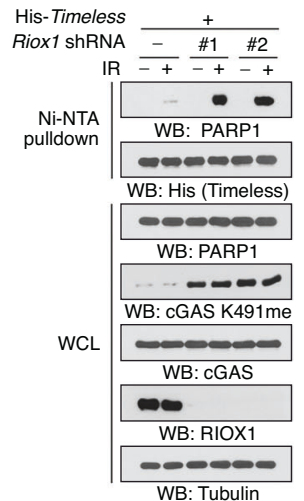

j

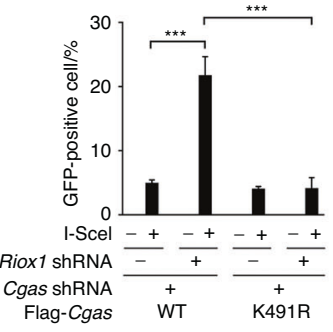

Fig. 4 RIOX1-mediated demethylation of cGAS K491me facilitates cGAS binding to PAR. a-d, f, g, i Immunoblot analysis was performed. a Equal amounts of biotin-PAR were incubated with bacterially purified WT His-CGAS or the indicated mutant protein, and a streptavidin pulldown assay was performed. b The DNA binding site, catalytic domain and putative PAR binding motif in the human cGAS structure (PDB ID: 6CTA) are shown. The basic and hydrophobic residues in the putative PAR binding motif are shown in yellow. c Equal amounts of biotinPAR were incubated with lysates of untreated or Riox1-depleted 32D Cl3 cells (upper panel). Equal amounts of biotin-PAR were incubated with lysates of AML12 or 32D Cl3 cells (bottom panel). The cell lysate input was used for normalization of the cGAS expression level. A streptavidin pulldown assay was performed. d 32D Cl3 cells expressing Riox1 shRNA or Flag-Cgas were treated with 10Gy ionizing radiation. Immunoprecipitation was performed. The immunoprecipitates were treated with an excess of purified PARG protein before being subjected to immunoblot analysis. e 32D Cl3 cells expressing Riox1 shRNA were transfected with an I-Scel expression vector. ChIP-PCR was performed. f 32D Cl3 cells expressing Riox 1 shRNA or His-Timeless were treated with $10 \mathrm{~Gy}$ ionizing radiation. A Ni-NTA pulldown assay was performed. The precipitates were treated with an excess of purified PARG protein before being subjected to immunoblot analysis. $\mathbf{g} 32 \mathrm{D} \mathrm{Cl} 3 \mathrm{cells}$ expressing Riox1 shRNA, WT Flag-Cgas or Flag-Cgas K491R were treated with $10 \mathrm{~Gy}$ ionizing radiation. Immunoprecipitation was performed. The immunoprecipitates were treated with an excess of purified PARG protein before being subjected to immunoblot analysis. 32D Cl3 cells expressing Riox1 shRNA, WT Flag-Cgas or Flag-Cgas K491R were transfected with an I-Scel expression vector. ChIP-PCR was performed 30 $\mathrm{h}$ after transfection $\mathbf{h}$. HR pathway activity was measured $72 \mathrm{~h}$ after transfection $\mathbf{j}$. The data are shown as the mean \pm SD of three independent assays. ${ }^{* *} P<0.001$. i 32D Cl3 cells expressing Riox1 shRNA, WT Flag-Cgas, Flag-Cgas K491R, or His-Timeless were treated with $10 \mathrm{~Gy}$ ionizing radiation. A Ni-NTA pulldown assay and immunoprecipitation were performed. The immunoprecipitates were treated with an excess of purified PARG protein before being subjected to immunoblot analysis

the biotin-PAR-mediated pulldown of purified K491me-bearing cGAS proteins (Fig. 5e). Consistent with this finding, reconstitution of SGF29 expression with the SGF29 D194A/D196A mutant restored the formation of the PARP1/cGAS complex, disrupted the formation of the PARP1/Timeless complex, blocked the recruitment of Timeless to DNA break sites, and limited HR repair to a low level even in RIOX1-depleted cells (Fig. $5 f-h$ ). These results suggest that SGF29 is involved in RIOX1-modulated HR repair by 


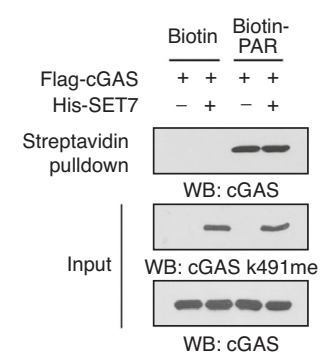

d

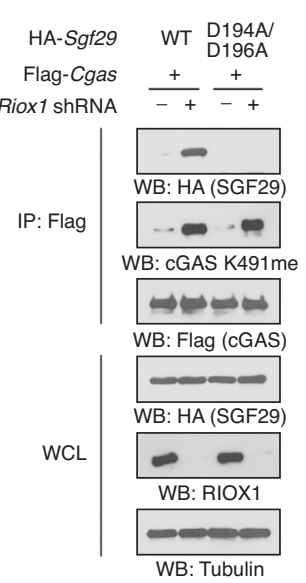

e

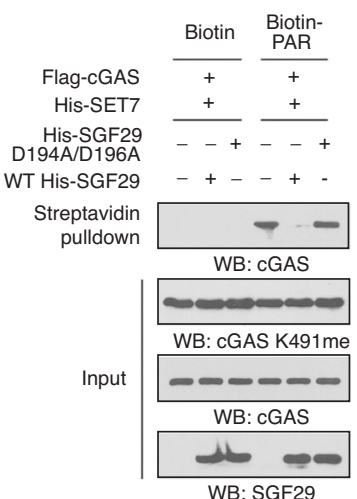

b

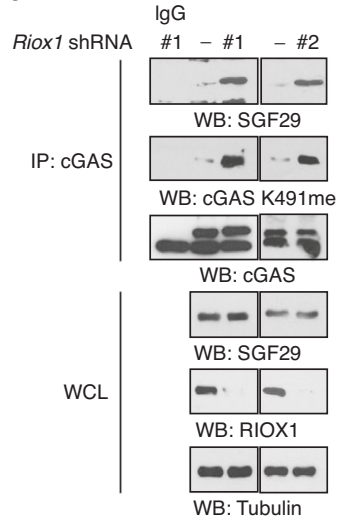

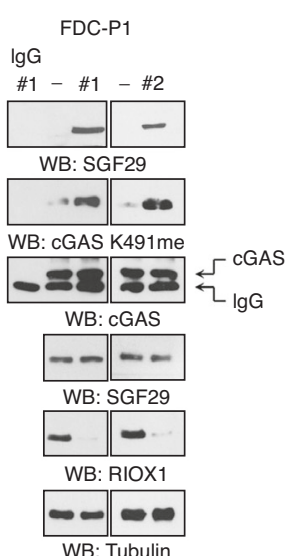

C

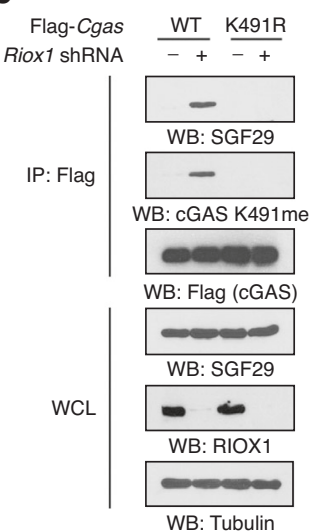

f

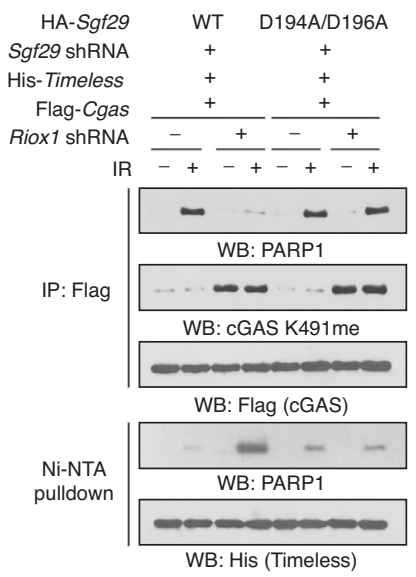

g

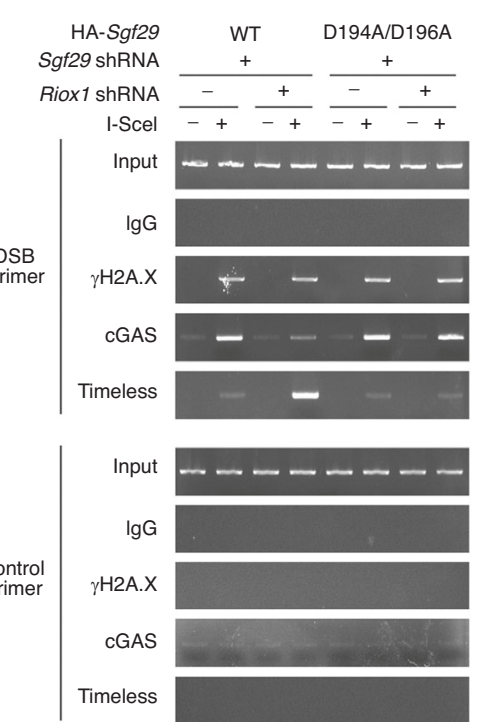

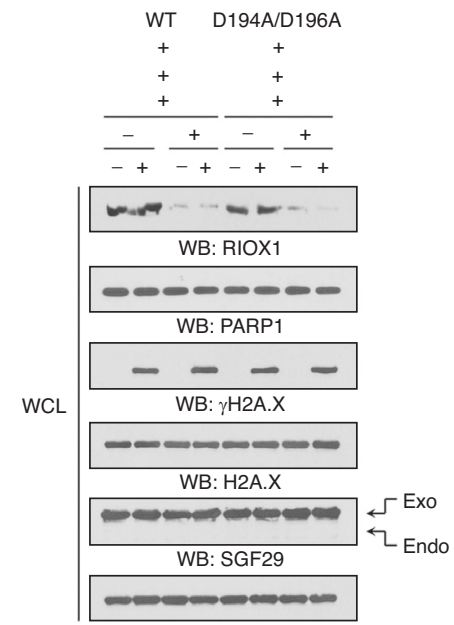

WB: Tubulin

h

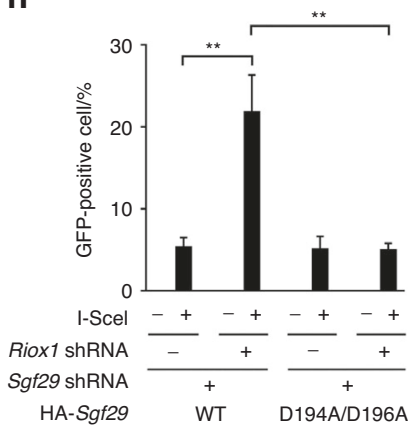

Fig. 5 SGF29 binds to K491me-bearing CGAS and prevents CGAS from binding to PAR. a-f Immunoblot analysis was performed. a Purified Flag-cGAS protein was incubated with purified His-SET7 protein for an in vitro methylation assay and was then incubated with an equal amount of biotin-PAR. A streptavidin pulldown assay was performed. b 32D Cl3 and FDC-P1 cells were transduced with Riox 1 shRNAs. Coimmunoprecipitation was performed using 32D Cl3 and FDC-P1 cell lysates. c 32D Cl3 cells were transduced with Riox1 shRNA, WT FlagCgas, or Flag-Cgas K491R. Immunoprecipitation was performed. d 32D Cl3 cells were transduced with Riox1 shRNA, Flag-Cgas, WT HA-Sgf29 or HA-Sgf29 D194A/D196A. Immunoprecipitation was performed. e Purified Flag-cGAS protein was incubated with purified His-SET7 protein for an in vitro methylation assay, incubated with purified WT or the indicated mutant SGF29 protein, and then incubated with an equal amount of biotin-PAR. A streptavidin pulldown assay was performed. f 32D Cl3 cells expressing Riox1 shRNA, Flag-Cgas, His-Timeless, Sgf29 shRNA, WT HASgf29, or HA-Sgf29 D194A/D196A were treated with $10 \mathrm{~Gy}$ ionizing radiation. A Ni-NTA pulldown assay and immunoprecipitation were performed. The immunoprecipitates were treated with an excess of purified PARG protein before being subjected to immunoblot analysis. The Sgf29 shRNA targeted the noncoding region. g, h 32D Cl3 cells expressing Riox1 shRNA, Sgf29 shRNA, WT HA-Sgf29, or HA-Sgf29 D194A/D196A were transfected with an I-Scel expression vector. ChIP-PCR was performed $30 \mathrm{~h}$ after transfection g. HR pathway activity was measured $72 \mathrm{~h}$ after transfection $\mathbf{h}$. The data are shown as the mean \pm SD of three independent assays. ${ }^{*} P<0.01$ 
interacting with K491me-bearing cGAS to prevent cGAS from binding to PAR.

Human RIOX1 modulates HR repair by regulating the methylation of the corresponding human cGAS K506

Human CGAS contains a conserved K506 site corresponding to K491 in mouse cGAS (Fig. S3B). To further investigate whether the RIOX1mediated mechanism is species- or cell type-specific, we validated our aforementioned findings in human oral keratinocytes (HOKs). As expected, knockdown of RIOX1 by shRNAs enhanced HR repair after exposure to ionizing radiation (Fig. S5A). Human CGAS was capable of interacting with RIOX1 and SET7 (Fig. S5B and S5C). By using a validated antibody recognizing human cGAS K506me (Fig. S5D), we found that loss of RIOX1 largely increased the level of cGAS K506me and induced the association between CGAS and SGF29 in HOKs (Fig. S5E and S5F). In contrast, the basal cGAS K506me was totally abolished by knockdown of SET7 (Fig. S5G). Furthermore, the cGAS K506R or SGF29 D194A/D196 A mutation promoted the binding of CGAS with PARP1 and abrogated the interaction between PARP1 and Timeless (Fig. S5H-S5J). These results suggest that the RIOX1-guided regulation of $\mathrm{HR}$ repair is conserved between human and mouse cells.

RIOX1-modulated cGAS K491me regulates the radiosensitivity of bone marrow cells

To determine the biological impact of cGAS K491me and the cGAS/SGF29 interaction on bone marrow cells, we knocked down endogenous CGAS or SGF29 in RIOX1-intact or RIOX1-depleted 32D Cl3 and FDC-P1 cells and exogenously expressed the cGAS K491R or SGF29 D194A/D196A mutant. Although loss of RIOX1 substantially increased cell viability and reduced cell death after irradiation, these effects were largely abolished by the cGAS K491R or SGF29 D194A/D196A mutation (Fig. 6a-d). Together, these results suggest that RIOX1-modulated cGAS K491me regulates the radiosensitivity of bone marrow cells.

\section{DISCUSSION}

lonizing radiation-induced DNA damage is cytotoxic and becomes lethal when it is not repaired properly and effectively. ${ }^{25}$ RIOX 1 was previously identified in a proteomic screen as a binding partner of the transcription factor Osterix. ${ }^{9}$ Ablation of RIOX1 promoted bone formation in mice, leading to increased bone mass and density. ${ }^{26}$ In the present study, we demonstrated that both bone marrow cells isolated from RIOX1 knockout mice and Riox 1 shRNA-treated bone marrow-derived cell lines showed increased viability after exposure to ionizing radiation. Accordingly, the efficiency of HR repair, rather than NHEJ repair, was markedly enhanced in RIOX1depleted cells. Therefore, our data highlight a novel role of RIOX1 in regulating the radiosensitivity of bone marrow cells.

Lysine methylation and demethylation are dynamic cyclical processes, and a well-balanced protein lysine methylation status is a prerequisite for diverse biological processes, including DNA repair. ${ }^{22}$ In this study, by using in vitro methylation/demethylation assays, we demonstrated that cGAS K491 can be monomethylated by SET7 and that this methylation is removed by RIOX1. Although SET7 can catalyze the methylation of multiple nonhistone proteins, ${ }^{27}$ to the best of our knowledge, cGAS is the first nonhistone demethylation substrate discovered for RIOX1. More importantly, we demonstrated that RIOX1 is highly expressed in bone marrow tissues and bone marrow-derived cell lines, accompanied by a low cGAS K491me level. In contrast, the expression level of SET7 fluctuates only minimally among different tissues and organs. Therefore, the present data suggest that the expression of RIOX1 is the dominant factor controlling the cGAS K491me level.

cGAS is a cytosolic DNA sensor. ${ }^{28}$ Either exogenous or endogenous DNA can trigger cGAS-dependent production of CGAMP, which in turn activates STING to elicit innate immune responses. ${ }^{19}$ Recently, cGAS was found to translocate into the nucleus in response to ionizing radiation. Independent of its nucleotidyltransferase activity, nuclear cGAS binds to the PAR chain of PARP1 and competitively inhibits PARP1-mediated recruitment of Timeless to DNA damage sites, retarding the process of HR repair ${ }^{18}$. In this study, we further deciphered how the interaction between cGAS and PAR is regulated by protein methylation. We found that the 483-497 aa region at the C-terminus of the cGAS protein, which contains the K491 methylation site, is responsible for cGAS binding with PAR. The methyllysine reader protein SGF29 binds to K491me-bearing cGAS and masks, at least partially, the PAR binding motif, thereby sequestering CGAS and preventing it from interacting with PAR (Fig. 6e). Therefore, our current data add to the current knowledge of the nonimmune function of CGAS in DNA repair.

As previously reported, JMJD-containing demethylases, such as JMJD1A, JHDM2A and JMJD5, modulate DNA repair efficiency through genome-wide histone modifications. ${ }^{6-8}$ The present study demonstrates a novel RIOX1-mediated mechanism in which RIOX1 acts as a nonhistone demethylase to govern cGAS K491me and consequently modulates the HR repair machinery at DNA break sites. Furthermore, the correlation of high RIOX1 expression and low cGAS K491me and the increased survival rate of RIOX1-depleted bone marrow cells underscore the critical role of RIOX1 in the regulation of cellular radiosensitivity. The present data suggest that RIOX1 inhibitors might be potential bone marrow-protective agents during radiotherapy.

\section{MATERIALS AND METHODS}

Materials

The rabbit polyclonal anti-cGAS K491me antibody was produced by Boer Biotechnology (Chengdu, China). The mouse cGAS K491me peptide was synthesized and injected into rabbits. An affinity column with bound nonmodified peptide was used to collect and purify rabbit serum, excluding antibodies targeting nonmethylated cGAS. The antibody was then purified using an affinity column linked with bound cGAS K491me peptide. A rabbit polyclonal antibody recognizing human cGAS K506me was produced following the same protocol.

Antibodies recognizing cGAS, Tubulin, GST, His, SET1A, SET1B, SET7, MLL1, MLL3, YH2A.X and SMYD3 were obtained from Cell Signaling Technology. Antibodies recognizing RIOX2 were purchased from Atlas Antibodies. Antibodies recognizing RIOX1, ASH1L, PARP1, MLL2, SGF29, Timeless, NSD1, NSD2, H2A.X, CD11b, CD11a, CD34, Thy-1, and SMYD2 were purchased from Abcam. The antibody against Flag, antiFlag M2 agarose beads, streptavidin-conjugated agarose beads, biotin, bovine serum albumin and poly(ADP-ribose) glycohydrolase (PARG) recombinant protein were purchased from Sigma. Horseradish peroxidase-conjugated goat anti-mouse and anti-rabbit secondary antibodies were purchased from Thermo Fisher Scientific. Biotin (terminal)-PAR Polymer was purchased from Trevigen.

\section{DNA constructs and mutagenesis}

The PCR-amplified mouse Cgas, Riox1, Riox2, Set7, Timeless and Sgf29 and human CGAS, SET7, Timeless and SGF29 sequences were subcloned into the indicated vectors. A QuikChange SiteDirected Mutagenesis Kit (Stratagene, La Jolla, CA) was used to generate the mutant constructs.

The following shRNAs were used in this study: scrambled shRNA, GCTTCTAACACCGGAGGT CTT; mouse Riox1 shRNA-1, TITTAATAAATCTGACGT (recognizing a noncoding sequence); mouse Riox1 shRNA-2, TATCATATAACATGGTTGC; mouse Cgas shRNA, TITCAGAAG GCAATGTCAG (recognizing a noncoding sequence); mouse Set7 shRNA-1, AACAGTATTAGGTCCAACT; mouse Set7 shRNA-2, AATCCGTCATCGTCCAGGT; mouse Sgf29 shRNA, TATTTGATCATAGGAACTC (recognizing a noncoding sequence); mouse $H 3 f 3 b$ shRNA, ACAAATGCAGTCTAGTCAG (recognizing a noncoding sequence); human CGAS shRNA, AGTTCTTACTGAAAAACAG (recognizing a noncoding sequence); and human SGF29 shRNA, ACGCAGG 
a

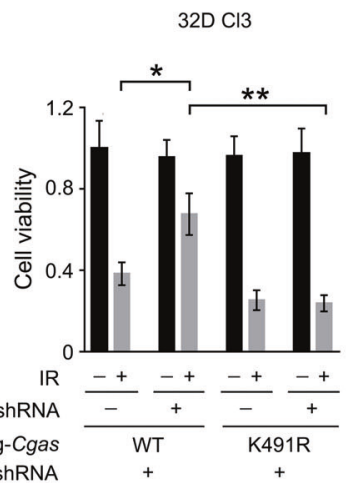

C

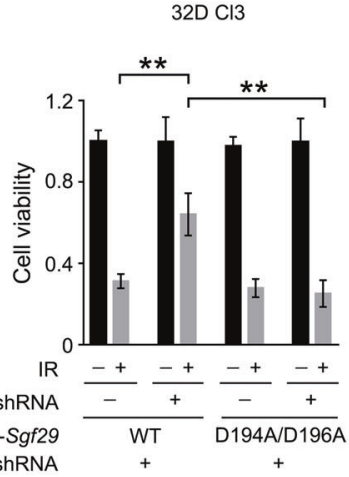

FDC-P1

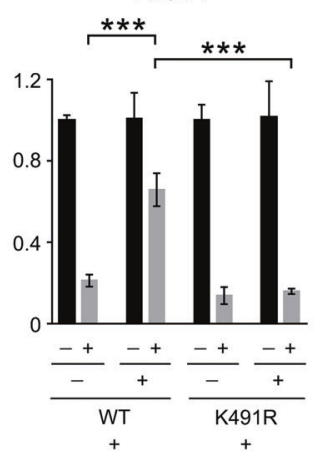

FDC-P1

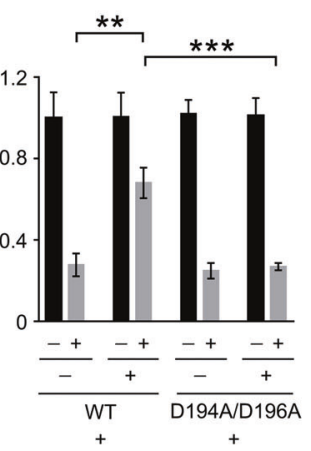

b

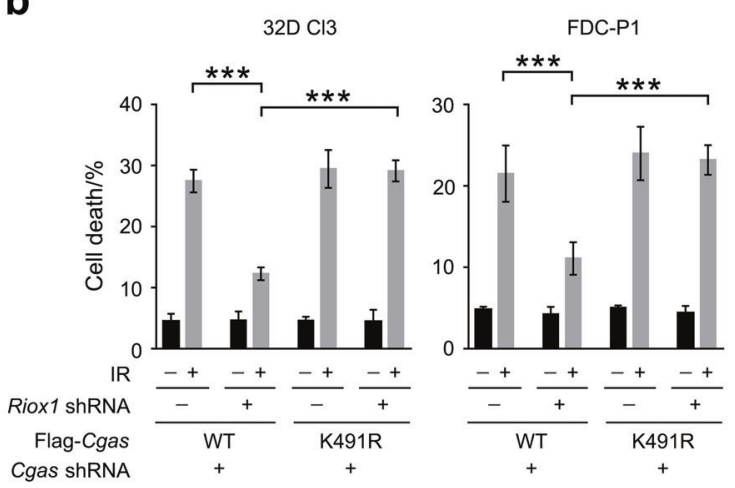

d
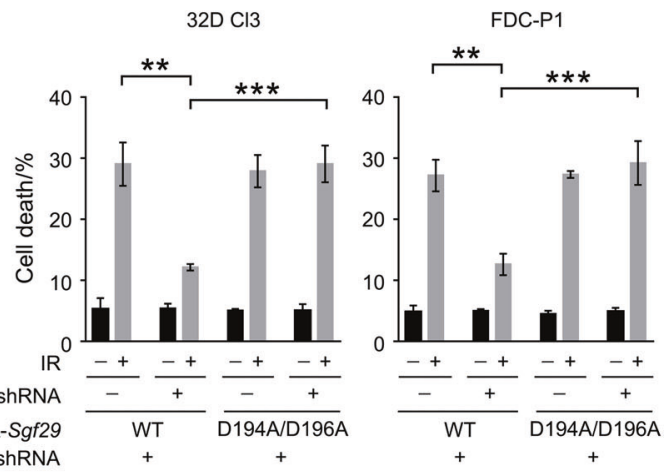

e
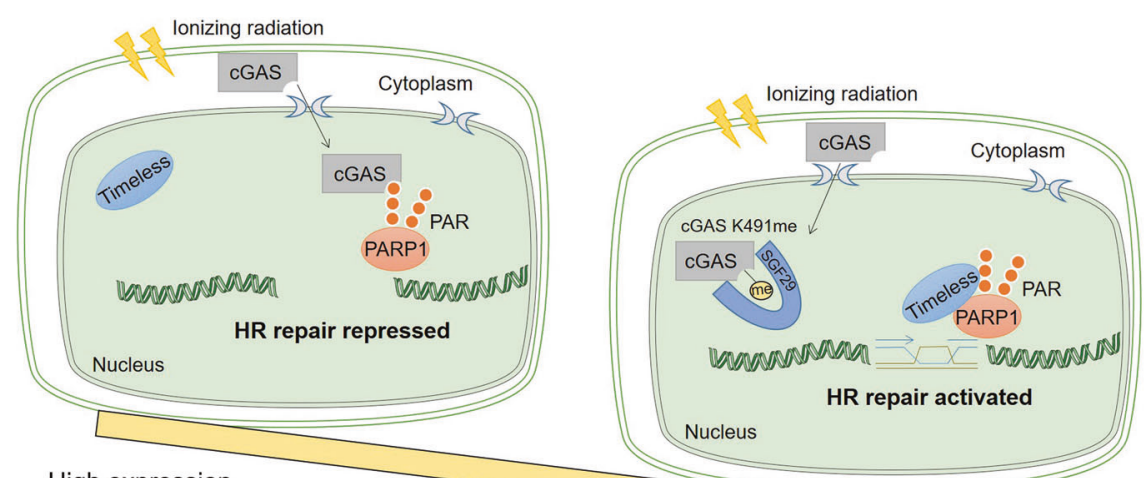

High expression

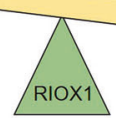

Low expression

Fig. 6 RIOX1-mediated CGAS K491me and the CGAS/SGF29 interaction regulate the radiosensitivity of bone marrow cells. a-d The data are shown as the mean \pm SD of three independent assays. ${ }^{*} P<0.05 ; * * P<0.01 ;{ }^{* * *} P<0.001$. a, b $32 \mathrm{D}$ Cl3 and FDC-P1 cells expressing Riox1 shRNA Cgas shRNA, WT Flag-Cgas, or Flag-Cgas K491R were treated with $10 \mathrm{~Gy}$ ionizing radiation. Cell viability a and cell death $\mathbf{b}$ were evaluated $48 \mathrm{~h}$ after irradiation. Cell viability data were normalized to the untreated group. c, d 32D Cl3 or FDC-P1 cells expressing Riox 1 shRNA, Sgf29 shRNA, WT HA-Sgf29, or HA-Sgf29 D194A/D196A were treated with $10 \mathrm{~Gy}$ ionizing radiation. Cell viability $\mathbf{c}$ and cell death $\mathbf{d}$ were evaluated $48 \mathrm{~h}$ after irradiation. Cell viability data were normalized to the untreated group. e Schematic of RIOX1-mediated repression of HR repair

TCTGTGATCATC (recognizing a noncoding sequence). Other shRNAs used in this study were obtained from Sigma.

Cell culture and stable cell line establishment

32D Cl3 and FDC-P1 cells were maintained in RPMI 1640 medium containing $10 \%$ fetal bovine serum (FBS). C2C12 and NIH/3T3 cells were cultured in Dulbecco's modified Eagle's medium (DMEM) containing $10 \%$ FBS. AML12 cells were grown in DMEM:F12 medium supplemented with $10 \%$ FBS. MLE 12 cells were maintained in HITES medium supplemented with $2 \%$ FBS. HOKs were obtained from ScienCell (Carlsbad, CA) and were maintained in Oral Keratinocyte Medium (Carlsbad, CA) supplemented with $10 \%$ fetal bovine serum.
To generate stable cell lines with depletion of gene expression, cells were transfected with lentiviral vectors carrying shRNAs and subsequently selected with puromycin. To generate stable cell lines with gene overexpression, cells with silencing of endogenous gene expression were transduced with lentiviral vectors carrying the indicated gene sequence and were then selected with hygromycin.

Mice irradiation and bone marrow cell isolation Irradiation of six-week-old C57BL/6 mice was performed with a ${ }^{137}$ Cs gamma-ray source. A single dose of radiation was administered at $1 \mathrm{~Gy}$ per min. Mice were then sacrificed, and tibiae were harvested. An EasySep ${ }^{\text {TM }}$ Mouse Hematopoietic 
Progenitor Cell Isolation Kit (STEMCELL Technologies) was used to isolate hematopoietic progenitor cells; Dynabeads ${ }^{\text {TM }}$ Mouse Pan B (Thermo Fisher Scientific) was utilized to isolate B cells; and an EasySep $^{\mathrm{TM}}$ Mouse Neutrophil Enrichment Kit (STEMCELL Technologies) was utilized to isolate neutrophils. Animals were treated according to the relevant institutional and national guidelines and regulations. The use of animals was approved by the institutional review board of West China Hospital of Stomatology.

Cell viability assay

A Cell Counting Kit 8 (WST-8/CCK-8) was purchased from Abcam, and the assay was performed following the manufacturer's protocol.

Cell death assay

Cell death was assessed by trypan blue staining as reported previously. $^{29}$

Generation of RIOX1 knockout mice

RIOX1 knockout mice were generated via CRISPR/Cas9 gene editing by GemPharmatech.

Protein expression in bacteria and protein purification

BL21(DE3) cells expressing cGAS, cGAS, cGAS, RIOX1, SET7, SGF29 and the corresponding mutant proteins were maintained in LB medium and induced with isopropyl $\beta$-D-1-thiogalactopyranoside (IPTG) for $16 \mathrm{~h}$ at $30^{\circ} \mathrm{C}$ before lysis via sonication. Purification of the tagged proteins was performed as reported previously. ${ }^{30}$

Analyses of DNA repair efficiency

NHEJ and HR repair were analyzed based on previously reported methods $^{13}$, and a schematic of this assay is shown in Fig. S1G. In brief, 32D Cl3 or FDC-P1 cells were transfected with the DR-GFP plasmid, which was unable to express DR-GFP because of the I-Scel site. Expression of I-Scel induced a DSB in the DR-GFP locus. The PCR products were amplified with primers targeting the sequences flanking the I-Scel site and were then double digested with the enzymes I-Scel and Bcgl. NHEJ repair caused loss of the I-Scel site and made the PCR product resistant to double digestion with I-Scel and Bcgl, producing a $0.65-\mathrm{kb}$ PCR band. HR repair caused the I-Scel-digested sequence to be replaced with a $\mathrm{Bcgl}$ cut site, restoring the expression of GFP.

DR-GFP-expressing cells were immediately cotransfected with a vector expressing I-Scel. After transfection for $36 \mathrm{~h}$, to inhibit DNA repair and promote the accumulation of I-Scel-mediated DSBs, the cells were treated with NU7441 $\left(1 \mu \mathrm{mol} \cdot \mathrm{L}^{-1}\right)$ and KU55933 $\left(10 \mu \mathrm{mol} \cdot \mathrm{L}^{-1}\right)$ for $12 \mathrm{~h}$ and were then exposed to ionizing radiation. To measure the NHEJ repair efficiency, cells were synchronized in G1 phase by double thymidine block, and genomic DNA was extracted $1 \mathrm{~h}$ after ionizing radiation to quantify the $0.65-\mathrm{kb}$ PCR product. To measure the HR repair efficiency, cells were stained with Hoechst $72 \mathrm{~h}$ after ionizing radiation. The numbers of GFP-expressing and Hoechst-positive cells were counted under a fluorescence microscope to calculate the ratio of GFP-positive cells to total cells. At least 10 fields, each containing more than 100 cells, were counted. To measure the efficiency of I-Scel cleavage, genomic DNA was extracted immediately after irradiation. The amount of $0.65-\mathrm{kb}$ PCR product generated from the naive, I-Scel-uncut DNA was used as an indirect reference value for I-Scel-induced DSBs. $\beta$-Actin was used as the internal control. The primer sequences were as follows: DRGFP-F, 5-CTGCTAACCATGTTCATGCC-3; DR-GFP-R, 5-AAGTCGTGCT GCTTCATGTG-3; $\beta$-actin-F, 5- GACAGGATGCAGAAGGAGATT ACTG; $\beta$-actin-R, CTCAGGAGGAGCAATGATCTTGAT.

\section{ChIP-PCR assay}

As reported previously, an Upstate Biotechnology kit was used to perform the ChIP-PCR assay. ${ }^{30}$ The specific primers used for PCR were designed to target the sequence $0.2 \mathrm{~kb}$ away from the I-Scel- induced DSBs: 5-GATCAGGCAGAGCAGGAACC-3 (forward) and 5-GAACAGCTCCTCGCC CTTGC-3 (reverse).

Comet assay

Total bone marrow cells were isolated from Riox 1 knockout mice or WT mice and subjected to $10 \mathrm{~Gy}$ ionizing radiation. The comet assay was performed using a Single Cell Gel Electrophoresis Assay/ Comet Assay (R\&D Systems) following the manufacturer's protocol.

In vitro methylation assay

Purified SET7 protein $(500 \mathrm{ng}$ ) and purified cGAS protein (200 ng) were incubated in $100 \mu \mathrm{L}$ of methylation buffer at $30^{\circ} \mathrm{C}$ for $1 \mathrm{~h}$ according to a previously reported method. ${ }^{31}$ SDS loading buffer was added to stop the reaction, and immunoblot analysis was then conducted.

In vitro demethylation assay

Purified RIOX1 protein (500 ng) and purified cGAS protein (200 ng) were incubated with demethylase reaction buffer at $37^{\circ} \mathrm{C}$ for $1 \mathrm{~h}$, as mentioned previously. ${ }^{32}$ SDS loading buffer was added to stop the reaction, and immunoblot analysis was then conducted.

cGAS activity assay

cGAS activity was detected by using a Transcreener cGAMP cGAS TR-FRET Assay Kit (BellBrook Labs) following the manufacturer's instructions.

Diarrhea severity assessment

Animals were subjected to irradiation for 5 days, and diarrhea severity was recorded according to a previously defined grading system ${ }^{33}$.

Statistical analysis

Unless specifically indicated, significant differences were analyzed by two-tailed unpaired Student's $t$ test. Unless otherwise specified, all data are presented as the mean $\pm S D$ of three independent experiments/samples.

\section{ACKNOWLEDGEMENTS}

This work was supported by the National Natural Science Foundation of China (Grants 81872218 (R.L.) and 81772692 (J.L.)); CAMS Innovation Fund for Medical Sciences (CIFMS, 2019-I2M-5-004 (Q.C.)); Sichuan Science \& Technology Program (2020YFSY0009 (Q.C.)); Research Fund from West China School/Hospital of Stomatology Sichuan University (RCDWJS2020-8 (R.L.)); the Scientific Research Program for Young Talents of China National Nuclear Corporation (2020CNNC74 (J.L.)); Research Fund from the Science \& Technology Department of Sichuan Province (2019YJ0371 (J.L.)); Research Fund from Chengdu Medical College (CYTD18-02 (J.L.)); Research Fund from Suzhou University (KJS1962 (J.L.)); and a National Research Foundation of Korea (NRF) grant funded by the Korean government (MSIT) (2020R1C1C1011350).

\section{AUTHOR CONTRIBUTIONS}

Y.C., Q.C., and R.L. conceived and designed the study; Y.X., J.L., T.H., X.L., C.Y., Y.H., L.J., Y.C., S.M.J., and J-H.L. performed the experiments; Y.C., C.Y. and R.L. drafted the manuscript.

\section{ADDITIONAL INFORMATION}

Supplementary information The online version contains supplementary material available at https://doi.org/10.1038/s41413-022-00194-0.

Competing interests: The authors declare no competing interests.

\section{REFERENCES}

1. Dubois, A. \& Walker, R. Prospects for management of gastrointestinal injury associated with the acute radiation syndrome. Gastroenterology 95, 500-507 (1988).

2. Kim, Y., Barshishat-Kupper, M., McCart, E., Mueller, G. \& Day, R. Bone marrow protein oxidation in response to ionizing radiation in $\mathrm{C} 57 \mathrm{BL} / 6 \mathrm{~J}$ mice. Proteomes $\mathbf{2}$, 291-302 (2014). 
3. Nickoloff, J. A., Sharma, N. \& Taylor, L. Clustered DNA double-strand breaks: biological effects and relevance to cancer radiotherapy. Genes 11, 99 (2020).

4. Cann, K. L. \& Hicks, G. G. Regulation of the cellular DNA double-strand break response. Biochem. Cell Biol. 85, 663-674 (2007).

5. Zhao, X. et al. Cell cycle-dependent control of homologous recombination. Acta Biochim. Biophys. Sin. 49, 655-668 (2017).

6. Oh, S., Shin, S. \& Janknecht, R. The small members of the JMJD protein family: enzymatic jewels or jinxes? Biochimica et. Biophysica Acta Rev. Cancer 1871, 406-418 (2019).

7. Zhang, A. L. et al. Histone demethylase JHDM2A regulates $\mathrm{H} 3 \mathrm{~K} 9$ dimethylation in response to arsenic-induced DNA damage and repair in normal human liver cells. J. Appl. Toxicol.: JAT 41, 650 (2021).

8. Amendola, P. G. et al. JMJD-5/KDM8 regulates $\mathrm{H} 3 \mathrm{~K} 36 \mathrm{me} 2$ and is required for late steps of homologous recombination and genome integrity. PLoS Genet. 13, e1006632 (2017).

9. Sinha, K., Yasuda, H., Coombes, M., Dent, S. \& de Crombrugghe, B. Regulation of the osteoblast-specific transcription factor Osterix by NO66, a Jumonji family histone demethylase. EMBO J. 29, 68-79 (2010).

10. Guchhait, P., Tosi, M. F., Smith, C. W. \& Chakaraborty, A. The murine myeloid cell line $32 \mathrm{Dcl} 3$ as a model system for studying neutrophil functions. J. immunological methods 283, 195-204 (2003).

11. Allen, J. D. \& Adams, J. M. Enforced expression of Hlx homeobox gene prompts myeloid cell maturation and altered adherence properties of T cells. Blood $\mathbf{8 1}$, 3242-3251 (1993).

12. Kockler, Z., Osia, B., Lee, R., Musmaker, K. \& Malkova, A. Repair of DNA breaks by break-induced replication. Annu. Rev. Biochem. 90, 165-191 (2021).

13. Jiang, Y. et al. Local generation of fumarate promotes DNA repair through inhibition of histone H3 demethylation. Nat. Cell Biol. 17, 1158-1168 (2015).

14. Hou, H. \& Yu, H. Structural insights into histone lysine demethylation. Curr. Opin. Struct. Biol. 20, 739-748 (2010).

15. Hamiche, A. \& Shuaib, M. Chaperoning the histone H3 family. Biochim. Biophys. Acta 1819, 230-237 (2013).

16. Xia, Y. et al. RNF8 mediates histone $\mathrm{H} 3$ ubiquitylation and promotes glycolysis and tumorigenesis. J. Exp. Med. 214, 1843-1855 (2017).

17. Yang, W. et al. PKM2 phosphorylates histone $\mathrm{H} 3$ and promotes gene transcription and tumorigenesis. Cell 150, 685-696 (2012).

18. Liu, H. et al. Nuclear CGAS suppresses DNA repair and promotes tumorigenesis. Nature 563, 131-136 (2018).

19. Mackenzie, K. et al. cGAS surveillance of micronuclei links genome instability to innate immunity. Nature 548, 461-465 (2017).

20. Chen, Q., Sun, L. \& Chen, Z. J. Regulation and function of the CGAS-STING pathway of cytosolic DNA sensing. Nat. Immunol. 17, 1142-1149 (2016).

21. Pleschke, J. M., Kleczkowska, H. E., Strohm, M. \& Althaus, F. R. Poly(ADP-ribose) binds to specific domains in DNA damage checkpoint proteins. J. Biol. Chem. 275 40974-40980 (2000).

22. Völkel, P. \& Angrand, P.-O. The control of histone lysine methylation in epigenetic regulation. Biochimie 89, 1-20 (2007).
23. Huang, L.-Y., Hsu, D.-W. \& Pears, C.J. Methylation-directed acetylation of histone H3 regulates developmental sensitivity to histone deacetylase inhibition. Nucleic Acids Res. 49, 3781-3795 (2021).

24. Bian, C. et al. Sgf29 binds histone H3K4me2/3 and is required for SAGA complex recruitment and histone $\mathrm{H} 3$ acetylation. EMBO J. 30, 2829-2842 (2011).

25. Santivasi, W. \& Xia, F. lonizing radiation-induced DNA damage, response, and repair. Antioxid. Redox Signal. 21, 251-259 (2014).

26. Chen, Q. et al. Mesenchymal deletion of histone demethylase NO66 in mice promotes bone formation. J. Bone Miner. Res.: Off. J. Am. Soc. Bone Miner. Res. 30 1608-1617 (2015).

27. Pradhan, S., Chin, H., Estève, P. \& Jacobsen, S. SET7/9 mediated methylation of non-histone proteins in mammalian cells. Epigenetics 4, 383-387 (2009).

28. Sun, L., Wu, J., Du, F., Chen, X. \& Chen, Z. J. Cyclic GMP-AMP synthase is a cytosolic DNA sensor that activates the type I interferon pathway. Science 339, 786-791 (2013).

29. Crowley, L., Marfell, B., Christensen, M. \& Waterhouse, N. Measuring cell death by trypan blue uptake and light microscopy. Cold Spring Harb. Protoc. 2016, 7 (2016).

30. Xia, Y. et al. c-Jun downregulation by HDAC3-dependent transcriptional repression promotes osmotic stress-induced cell apoptosis. Mol. Cell 25, 219-232 (2007).

31. Amente, $\mathrm{S}$. et al. Identification of proteins interacting with the RNAPII FCP1 phosphatase: FCP1 forms a complex with arginine methyltransferase PRMT5 and it is a substrate for PRMT5-mediated methylation. FEBS Lett. 579, 683-689 (2005).

32. Whetstine, J. R. et al. Reversal of histone lysine trimethylation by the JMJD2 family of histone demethylases. Cell 125, 467-481 (2006).

33. Gibson, R. J., Bowen, J. M. \& Keefe, D. M. Palifermin reduces diarrhea and increases survival following irinotecan treatment in tumor-bearing DA rats. Int. J. Cancer 116, 464-470 (2005). Attribution 4.0 International License, which permits use, sharing,
distribution and reproduction in any medium or format, as long as you give adaptation, distribution and reproduction in any medium or format, as long as you give Commons license, and indicate if changes were made. The images or other third party material in this article are included in the article's Creative Commons license, unless indicated otherwise in a credit line to the material. If material is not included in the article's Creative Commons license and your intended use is not permitted by statutory regulation or exceeds the permitted use, you will need to obtain permission directly from the copyright holder. To view a copy of this license, visit http://creativecommons. org/licenses/by/4.0/.

(c) The Author(s) 2022 